\title{
Collective dynamics of liquid carbon tetrachloride studied by inelastic neutron scattering and computer simulation
}

Cite as: J. Chem. Phys. 96, 8477 (1992); https://doi.org/10.1063/1.462300

Submitted: 21 November 1991 . Accepted: 18 February 1992 . Published Online: 31 August 1998

M. García-Hernández, J. L. Martínez, F. J. Bermejo, A. Chahid, and E. Enciso

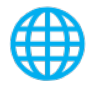

\section{Lock-in Amplifiers up to $600 \mathrm{MHz}$}




\title{
Collective dynamics of liquid carbon tetrachloride studied by inelastic neutron scattering and computer simulation
}

\author{
M. García-Hernández \\ Isis Pulsed Neutron Facility, Rutherford Appleton Laboratory, Chilton, Didcot, Oxon OX14 OQX, \\ Great Britain \\ J. L. Martínez \\ Institut Laue-Langevin, F-38042 Grenoble Cedex, France \\ F. J. Bermejo and A. Chahid \\ Instituto de Estructura de la Materia, Serrano 119, E-28006 Madrid, Spain \\ E. Enciso \\ Dpto. Quimica Fisica, U. Complutense de Madrid, E-28040 Madrid, Spain
}

(Received 21 November 1991; accepted 18 February 1992)

\begin{abstract}
An inelastic neutron scattering (INS) study of carbon tetrachloride $\left(\mathrm{CCl}_{4}\right)$ at two different temperatures within its liquid range is reported. The analysis of the spectra reveals the presence of a collective inelastic response throughout the explored $Q$-range $\left(0.3 \AA^{-1} \leqslant Q \leqslant 2.2\right.$ $\AA^{-1}$ ). The wave vector dependence of the excitation energies ("dispersion relations") is investigated by means of the analysis of the measured spectral intensities in terms of a phenomenological (viscoelastic) model. In order to help with the analysis of the experimental intensities a molecular dynamics study has been carried out. The analysis of the calculated dynamical factors is also performed within the same approximation and the results are tested by comparison with those stemming from the experimental observations.
\end{abstract}

\section{INTRODUCTION}

The question whether collective excitations at a molecular scale (involving distances of the order of a few tens of angstroms) are well defined entities in dense fluids has been extensively discussed during the last decade. ${ }^{1}$ Apart from the inherent theoretical problem of assigning a definite physical origin to these vibrations (exception made of those measurements performed in the hydrodynamic limit), the relatively scarce information obtained from experimental means, hindered any further progress in the field. Although in simple systems such as liquid metals or rare-gas fluids the situation has become to be understood on a quantitative basis, ${ }^{2.3}$ it remains at earlier states of development for fluids composed by nonspherical particles such as all the molecular liquids. In such a case, the difficulties arise from the need of separating the collective dynamics from the single-particle excitations (molecular rotations and translations), as well as from the fact that the interparticle potential substantially deviates from the idealized harmonic behavior. Although the first difficulty may be minimized if the experiments are performed on cold fluids, where the single-particle cross section mainly contributes to the resolution broadened elastic line, the problem of how the different intermolecular potentials are translated into collective dynamical properties at these length-scales, is still an open one.

On the other hand, apart from the case of liquid hydrogens, and in particular of liquid deuterium where the collective response has been shown to be a well defined entity up to relatively large momentum transfers, ${ }^{4}$ it seems clear nowadays that most of the observable collective features at the length and time scales of our interest will manifest themselves as strongly damped oscillations $\mathbf{s}^{5-8}$ which will render the analysis of the experimental intensities rather difficult unless some recourse is made to additional sources of data not amenable to experimentation with the present day means. ${ }^{9,10}$ In order to derive unambiguous information about the collective dynamics of these liquids from the analysis of the experimental intensities several steps have been taken. First of all, the low-frequency response embodying all the processes involving molecular diffusion and rotations (including coherent effects) has been analyzed in detail by means of neutron quasielastic scattering using different energy resolutions. ${ }^{11,12}$ From such a study it was possible to parameterize the relevant translational and rotational contributions to the total dynamic structure factor.

On the other hand, a set of computer experiments using a molecular dynamics (MD) algorithm was carried out in order to test the adequacy of the model employed for the analysis of neutron scattering intensities, and to extend the domain in reciprocal space to regions where it can be seen that the observed mode is basically a continuation to larger momentum-transfers of what is observable in regions nearer the hydrodynamic limit.

The purpose of the present work was to complement some previous studies on systems represented by a broad class of intermolecular potentials, ${ }^{5-8}$ with the study of a relatively simple liquid such as carbon tetrachloride $\left(\mathrm{CCl}_{4}\right)$ where the intermolecular interaction is overwhelmingly of Lennard-Jones-type (L-J) as revealed by quantitative comparisons of the liquid structure data with several model po- 
tentials. ${ }^{13}$ Furthermore, this liquid, which is considered to be the molecular analog to liquified rare-gases, represents an interesting case in order to ascertain whether the collective dynamics of L-J systems show some universal behavior dictated by the form of the interparticle potential, as it happens to be in the case of liquified classical rare-gases. ${ }^{14}$

The main difficulty which arises from the studies on collective phenomena in the class of liquids referred above, stems from the fact that only broad (strongly damped) features are observable within the kinematic range accessible to thermal neutrons using conventional inelastic neutron scattering (INS) spectroscopy, although the existence at larger length-scales of propagating short wavelength modes in these systems is now clearly established from neutron Brillouin studies. ${ }^{15}$

The absence of well resolved inelastic peaks will not imply that a collective response is not present in the data and, as a matter of fact, some previous studies on the same liquid using low incident energies ${ }^{11}$ have served as reference to test the presence of a collective (albeit overdamped, i.e., nonpropagating) mode at momentum-transfers achievable by conventional neutron inelastic scattering instrumentation.

The paper is structured as follows: Sec. II A is devoted to giving an account of the experimental conditions under which the spectra were recorded and of the various corrections that must be applied in order to render magnitudes amenable to comparison with the model predictions. Section II B summarizes the details of the MD simulations. The models used to interpret the experimental results are introduced in Sec. III, together with the computational strategy followed in the estimation of its parameters. Section IV includes a discussion on the results of the application of the models to both the experimental data and to the results of MD simulations. We conclude the paper with a resume of the main conclusions derived from our study.

\section{EXPERIMENTS}

\section{A. Neutron inelastic scattering}

A preliminary set of experiments was performed using the time-of-flight spectrometer IN4, located at one of the thermal beamlines of the Institut Laue Langevin (ILL), Grenoble. The purpose of these measurements was to estimate the dependence of the inelastic background upon the energy of the impinging neutrons, since it was clear that no clear inelastic response was found in previous low-energy measurements. ${ }^{11}$

A substantial enhancement of the inelastic background was found (after accounting for the different resolution functions involved) when using an incident energy of 10.85 $\mathrm{THz}$, whereas an experiment using $4.16 \mathrm{THz}$ failed to reveal the presence of substantial collective excitation.

A set of experiments at constant wave vector were performed using the triple-axis spectrometer IN8 at the ILL. ${ }^{16}$ The useful kinematic region for this class of experiments involves the transfers of rather high energy, at least 1.5 times the frequency corresponding to excitations of sonic modes due to the relatively large ratio of specific heats, ${ }^{17}$ for low $Q$ values (near the direct beam) so that a very restrictive colli- mation has to be used [30' (arc min) in pile, 20' between monochromator and sample, 10' between sample and analyzer, and $40^{\prime}$ between analyzer and detector]. All of the scans were recorded on constant- $Q$ mode with a fixed incident energy of $8.95 \mathrm{THz}$.

The monochromator and analyzer used the $(002)$ reflection of pyrolytic graphite, and the momentum-transfer range covered the region $0.3-2.2 \AA^{-1}$ including, therefore, the $Q$ value for which the structure factor reaches its first maximum $\left(Q \approx 1.3 \AA^{-1}\right)$. Both energy gain and loss sides were measured for all spectra covering the allowed full kinematic range.

The temperature was controlled using a standard "orange" cryostat with a regulation better than $0.3 \mathrm{~K}$. Experiments were performed at two different temperatures, $254 \mathrm{~K}$ (just above the melting point) and $310 \mathrm{~K}$. A cylindrical vacuum tank of $1 \mathrm{~m}$ diam was used to reduce the contribution coming from the diffuse scattering from the air. As a matter of fact, the evacuated tank provided a nearly complete reduction of the diffuse background. Several runs to estimate the instrumental background were carried out closing the beam coming into the analyzer by means of thick cadmium slabs.

The container is based in an original design by Söderstrom et $a .^{18}$ and has already been used in a number of previous experiments involving molecular liquids. ${ }^{5,6}$ Such a design has proven to reduce to a minimum the multiple scattering contribution since the beam is partly collimated by the cadmium-coated spacers. Results from the standard DISCUS (Ref. 19) code show that multiple scattering contributions amount to $<7 \%$ in all cases. Several measurements were carried out for the empty container at different $Q$-values and standard absorption corrections ${ }^{20}$ were performed prior to any can subtraction.

The instrumental resolution was also measured using a vanadium standard, giving a Gaussian shape of half-width at half-maximum (HWHM) of $\sim 0.4 \mathrm{THz}$. In order to account for the variation of the energy resolution along the accessible window, the width of the resolution function was evaluated by means of the RESCAL code ${ }^{21}$ at each energy-transfer value for every constant- $Q$ spectrum. Finally, to enable a direct comparison of spectra measured using instruments with large differences in energy resolution (i.e., IN6 and IN8 from ILL), the corrected spectra measured using the tripleaxis instrument were deconvolved from resolution effects by means of a code based upon a maximum-entropy algorithm previously tested for the study of excitations of this kind, ${ }^{5}$ as well as those of magnetic origin. ${ }^{22}$

Figure 1(a) shows a set of deconvolved spectra measured at IN8 as well as the corresponding quasielastic spectra (measured using IN6) which were converted into constant $Q$ by means of the INGRID code. ${ }^{23}$ The IN6 spectra were symmetrized in order to extend the energy-loss sides to facilitate the comparison.

As can be seen upon inspection of the graph, broad inelastic intensity distributions, not present in the quasielastic spectra, are clearly apparent in the triple-axis scans. On the other hand, the spectra corresponding to momentum-transfers below $0.7 \AA^{-1}$ show some evidence of the presence of 

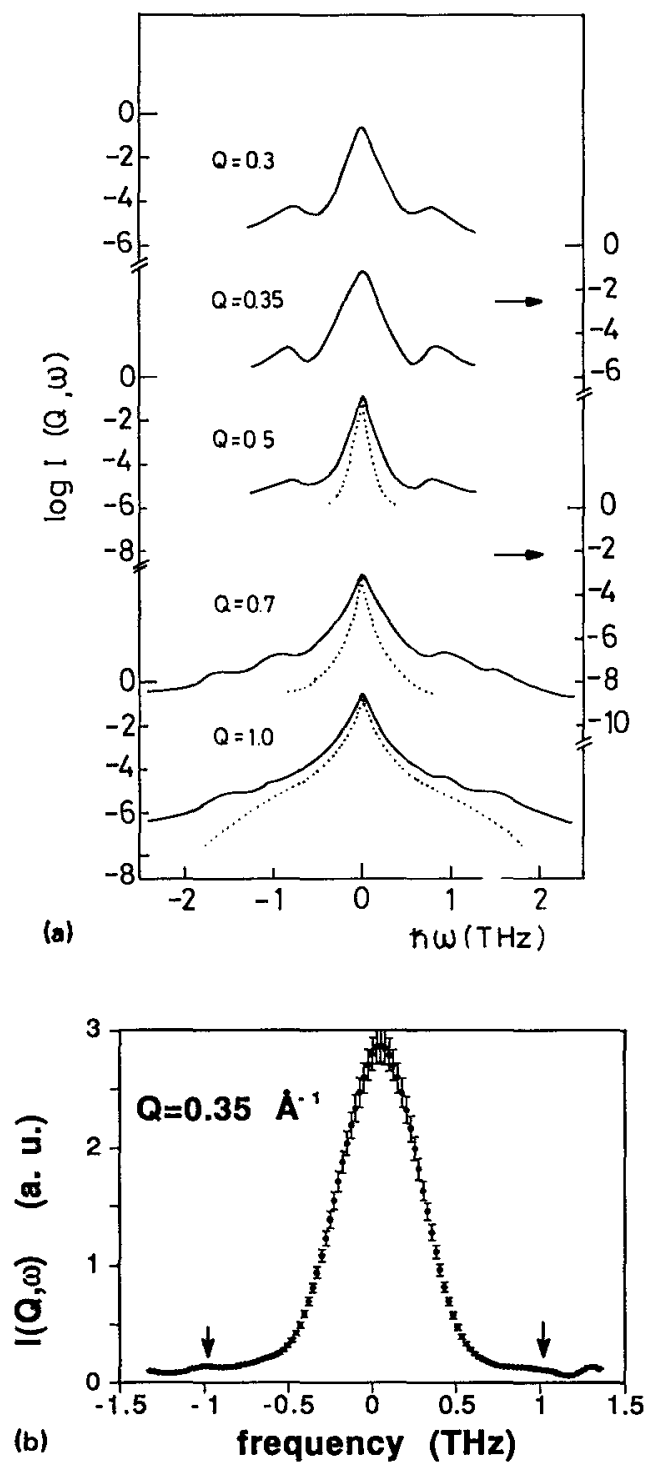

FIG. 1. (a) Logarithm of the spectral intensities measured at $T=254 \mathrm{~K}$ for the lowest values of the momentum transfer $Q$. The upper curves correspond to the triple-axis spectra deconvolved from resolution effects. The lower curves are constant- $Q$ quasielastic spectra (see text). (b) Sample fully corrected spectrum for a wave vector of $Q=0.35 \AA^{-1}$. Note that the blow-up at both sides arises from direct-beam contributions which are difficult to subtract.

weak inelastic side-peaks. As a matter of fact, Fig. 1(b) displays a spectrum corresponding to $Q=0.35 \AA^{-1}$ after corrections for sample absorption and multiple scattering. Clear inelastic features are seen at this, relatively low, momentum-transfer which become strongly damped at larger wave vectors. However, in order to assess whether such finite-frequency features appearing at wave vectors $<0.7 \AA^{-1}$ correspond to excitation peaks or are introduced as artifacts by the deconvolution procedure, it is required that they also manifest themselves in the structure factors computed by means of computer simulations and also in the analysis of the experimental (not-deconvolved) spectra in terms of a model function, as it is described in further sections.

\section{B. Computer experiments}

The standard program MDTETRA from the CCP5 Library, ${ }^{24}$ was used to generate the trajectories of 108 molecules subject to periodic cubic boundary conditions. Two simulations were carried out at two different thermodynamic states corresponding to temperatures around 260 and 293 $\mathrm{K}$, and densities of 1.66 and $1.58 \mathrm{~g} / \mathrm{cm}^{3}$, respectively. Both runs started from equilibration of a previous high-temperature configuration and the trajectories were followed for over a period of 90 and $80 \mathrm{ps}$ for the low and high temperature runs.

The equations of motion are solved by applying the Gear predictor-corrector integration method with a time step of 5 and $10 \mathrm{fs}$ for the low and high temperature runs. The center-of-mass motions were followed using a fifth-order algorithm and the Euler equations of motion, specified in quaternion form, were solved by a fourth-order algorithm. The intermolecular potential used here corresponds to a site-site Lennard-Jones as proposed by McDonald et al. ${ }^{13}$

$$
U\left(R_{12}, w_{1}, w_{2}\right)=\Sigma U_{\alpha \beta}\left(r_{\alpha \beta}\right),
$$

where $R_{12}$ is the vector joining the centers-of-mass of molecules 1 and 2 with relative orientations specified by $w_{i}$, and $r_{\alpha \beta}$ is the distance between atomic sites. The pair potential is then given in the usual form

$$
U_{\alpha \beta}\left(r_{\alpha \beta}\right)=4 \epsilon_{\alpha \beta}\left[\left(\sigma_{\alpha \beta} / r_{\alpha \beta}\right)^{12}-\left(\sigma_{\alpha \beta} / r_{\alpha \beta}\right)^{6}\right]
$$

and the interaction parameters for the carbon-carbon, carbon-chlorine, and chlorine-chlorine interactions were respectively, $\epsilon / k_{b}=51.2,72.4,102.4 \mathrm{~K}$ and $\sigma=0.460,0.405$, $0.305 \mathrm{~nm}^{-1}$. The potential was truncated with a site-to-site cutoff distance equivalent to one-half of the box width. Several time-dependent properties such as the velocity (linear and angular) correlation functions as well their associated memory functions and the scattering functions relevant for the analysis of quasielastic neutron scattering spectra have been computed, and an account was given in a previous paper. ${ }^{12}$

In order to ascertain whether any dispersive behavior occurs we have computed in the first instance the longitudinal current autocorrelation function, $J_{l}(Q, t)$, which is closely related to the intermediate scattering function, $F_{\text {coh }}(Q, t)$, through $^{25}$

$$
\frac{d^{2}}{d t^{2}} F(Q, t)=-Q^{2} J_{l}(Q, t)
$$

and, consequently, in the frequency domain it can be written

$$
\omega^{2} S(Q, \omega)=Q^{2} J_{l}(Q, \omega) .
$$

The use of such functions has some advantages when compared to the dynamic structure factor, $S(Q, \omega)$, namely, the oscillatory nature of the longitudinal current autocorrelation, $J_{l}(Q, t)$, at very short times renders spectra less affected by windowing effects than those stemming from the slower decaying intermediate scattering function, $F(Q, t)$. Furthermore, because of the $\omega^{2}$ factor in Eq. (3), the high frequency region of the spectra appears enhanced while the low frequency region (quasielastic) is depleted allowing, therefore, an easier tracking of the high frequency propagative modes. 




FIG. 2. Spectra of the normalized longitudinal current autocorrelation function at $T=264 \mathrm{~K}$ as a function of the frequency for the lowest six values of the momentum transfer accessible in our MD simulation. The low wave vector functions show a finite intercept value on the ordinate axis due to windowing effects.

This effect can be seen in Fig. 2, where a set of these functions is depicted for different $Q$-values. The fact that the computed currents at certain $Q$-values do not exhibit the proper limiting behavior when $\omega \rightarrow 0$ can be atributed to the use of a finite window function for the Fourier transformation into the frequency space. Such an effect is specially severe for low- $Q$ values where the intermediate scattering functions have not completely decayed. Figure 3 plots the value of the frequency, $\omega_{\max }$, for which the function $J_{l}(Q, \omega)$ reaches its maximum vs $Q$, for the entire $Q$-range explored at $T=260 \mathrm{~K}$. From this plot, it is readily apparent that the "dispersion" observed for $\omega_{\max }$ follows what it is expected for a propagative acoustic excitation, but now a shift towards higher frequencies occurs due to the $\omega^{2}$ factor in Eq. (4).

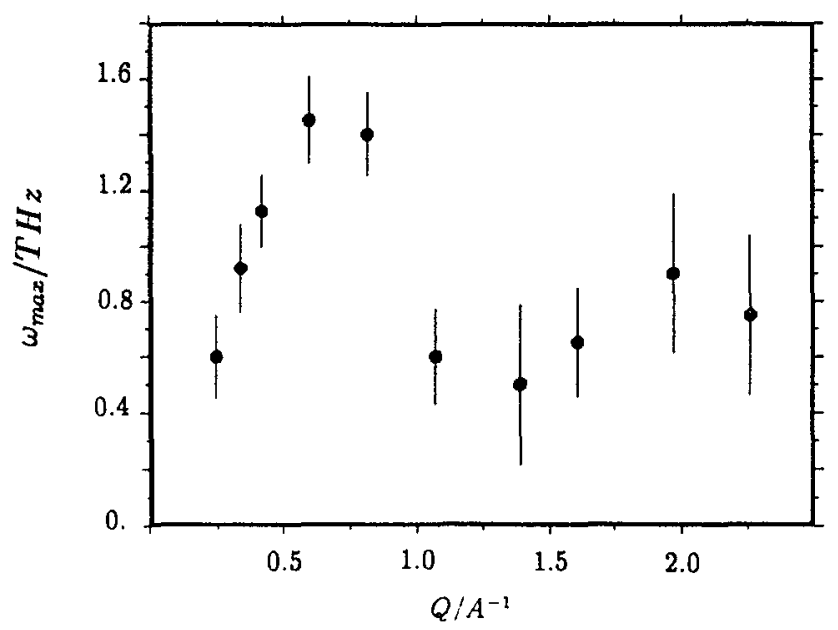

FIG. 3. Frequency of the maxima of the longitudinal current spectra for the lowest temperature MD simulation $(T=264 \mathrm{~K})$ as a function of the momentum transfer. Error bars have been estimated as the HWHM of the window function used for Fourier transforming the current autocorrelation function.

\section{DATA ANALYSIS AND RESULTS}

The measured double differential cross section can be written as a sum of quasielastic and inelastic contributions,

$$
\left(\frac{d^{2} \sigma}{d \Omega d \omega}\right)_{\text {total }}=\left(\frac{d^{2} \sigma}{d \Omega d \omega}\right)_{\text {qel }}+\left(\frac{d^{2} \sigma}{d \Omega d \omega}\right)_{\text {inel }} .
$$

The quasielastic contributions arise from coherent and incoherent effects and have been modeled from the parameterization described in Ref. 12. For the coherent part of the inelastic cross section we have used a model originally proposed by Lovesey for simple liquids ${ }^{26}$ and successfully used in similar analyses in molecular liquids. 5,6 The model rests upon the viscoelastic approximation and describes the collective inelastic contribution in terms of only two parameters, $\omega_{0}$ and $\omega_{l}$, related to the second and fourth moments of the dynamical structure factor, $S(Q, \omega)$, respectively. The model also includes a single relaxation time for which we adopt a Maxwellian form. This amounts to writing,

$$
\begin{aligned}
\left(\frac{d^{2} \sigma}{d \Omega d \omega}\right)_{\text {inel }}^{\mathrm{coh}}= & \frac{k_{f}}{k_{i}} B_{\mathrm{sc}}(Q)\left[\frac{1}{1-\exp \left(\frac{-h \omega}{k_{B} T}\right)}\right] \\
& \times \frac{\omega_{0}^{2}\left(\omega_{l}^{2}-\omega_{0}^{2}\right) \tau}{\left[\omega \tau\left(\omega^{2}-\omega_{l}^{2}\right)\right]^{2}+\left(\omega^{2}-\omega_{0}^{2}\right)^{2}} \\
\equiv & B_{\mathrm{sc}}(Q) S_{\mathrm{visc}}(Q, \omega),
\end{aligned}
$$

with $\tau$ given by

$$
\tau^{-1}=2 \sqrt{\left(\omega_{l}^{2}-\omega_{0}^{2}\right) / \pi}
$$

and $B_{\mathrm{sc}}(Q)$ is an amplitude factor.

It should be remarked at this point that due to the unavailability of closed-form expressions to calculate the relevant sum-rules for the two frequency moments for a molecular fluid, the derived parameters should be taken as "effective" ones, although for a fluid composed by highly symmetrical particles they are expected to show some trends in qualitative agreement with those expected for a monatomic liquid. In particular, they should reach a maximum around the Brillouin zone-boundary (halfway to the maximum of the static structure factor for the molecular centers) and a broad minimum at wave vectors where the structure factor shows its maximum.

Finally the described scattering law is convolved with the measured resolution function, $R(Q, \omega)$, and the calculated intensity is compared with that observed experimentally

$$
\begin{aligned}
I^{\text {model }}(Q, \omega)= & \frac{k_{f}}{k_{i}}\left[A_{\mathrm{sc}}(Q) S_{\mathrm{qel}}(Q, \omega)\right. \\
& \left.+B_{\mathrm{sc}}(Q) S_{\mathrm{visc}}(Q, \omega)\right] \otimes R(Q, \omega),
\end{aligned}
$$

where the symbol $\otimes$ means convolution.

The model scattering law used to analyze the simulation data differs from the one described above mainly in the absence of contributions stemming from rotational components since the simulation only gives information of centers of mass motions.

A set of center-of-mass $S(Q, \omega)$ coherent dynamic structure factor data obtained from the simulation at $260 \mathrm{~K}$ is 


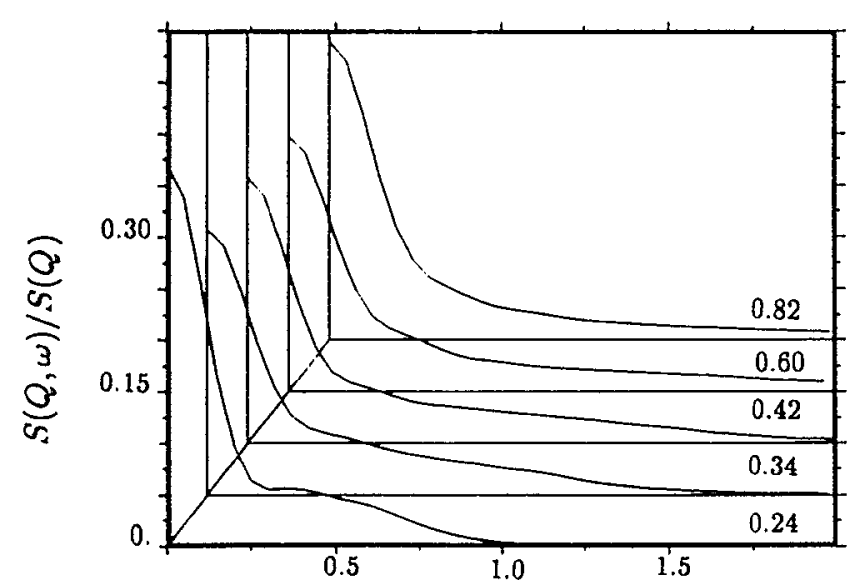

Frequency $/ \mathrm{THz}$

FIG. 4. Time Fourier transform of the normalized intermediate scattering function $F_{\text {coh }}(Q, t)$ for liquid $\mathrm{CCl}_{4}$ at $T=264 \mathrm{~K}$ for the five lowest values of momentum transfer accessible in our MD simulation.

shown in Fig. 4 as a function of the frequency $v(v=\omega / 2 \pi$, $\omega$ being the angular frequency). As it can be seen from the graph, well resolved Brillouin side peaks are not found in most of the $Q$-range accessible to our computer experiments,

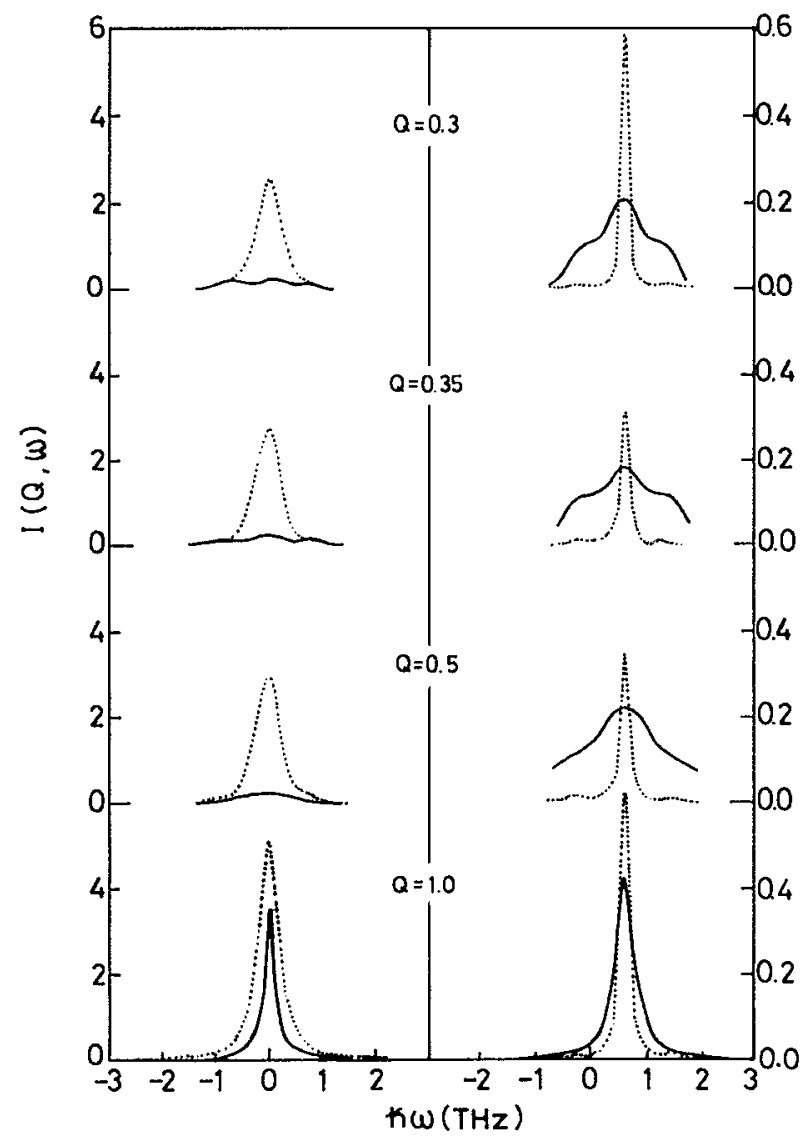

FIG. 5. On the left-hand side measured spectra for several representative values of momentum-transfers are shown as well as the collective response obtained from model fits to the total experimental intensities (including the quasielastic contribution), for $T=254 \mathrm{~K}$. The right-hand side depicts the deconvolved spectra as well as the collective component magnified to allow a comparison of the position of the side-peaks.


FIG. 6. (a) Square roots of the second and fourth normalized frequency moments derived from fits to the viscoelastic model. On the lower branch, open circles represent the $\omega_{0}$ values derived from fittings to the MD simulation $S(Q, \omega)$ and full circles represent those derived from fittings to the experimental spectra. On the upper branch, open squares represent the $\omega_{l}$ values derived from the fittings to the MD simulation spectra and full squares represent those derived from fittings to the measured intensities. The temperatures for the MD simulation experimental data are 264 and 254 $\mathrm{K}$, respectively. The slope of the solid line at low $Q$ values corresponds to the reported adiabatic sound velocity in Ref. 17 for $T=264 \mathrm{~K}$. (b) Same as (a) but for the temperatures of $T=310 \mathrm{~K}$ for the experimental data and $T=293 \mathrm{~K}$ for the simulation data.

an exception is made of the data corresponding to the lowest $Q$ spectrum $\left(Q=0.24 \AA^{-1}\right)$. Beyond that value the mode becomes heavily damped.

A nonlinear least-squares fitting program was assembled in order to estimate the values of the model parameters $\left(A_{\mathrm{sc}}, B_{\mathrm{sc}}, \omega_{0}\right.$, and $\left.\omega_{l}\right)$ which best fitted the experimental intensities for each momentum transfer value. In most cases, an initial search for the minimum of the objective function was conducted by means of a constrained version of the "simplex" algorithm and the resulting values of the model parameters were further optimized through a gradient search procedure.

Good fits were obtained in all cases and the fitted values of the parameters are given in Fig. 6 as well as the estimated variances which are indicated by means of a vertical bar.

Several comments are in order regarding the spectra plotted in Fig. 5. First and foremost, although the inelastic response is rather weak at low momentum-transfers, it is clearly present even in the lowest achieved value of $Q$. On the 
other hand, small inelastic peaks are clearly apparent up to $Q=0.5 \AA^{-1}$ whose position agrees with the one appearing in the deconvolved spectra as well as with the position of the shoulders of the fitted model functions. Finally, at wave vectors approaching the maxima of the static structure factor $S(Q)$, most of the intensity has merged into the central peak, which may be interpreted as a manifestation of the "simpleliquid" character of this material.

The spectra obtained from the MD simulation were analyzed following the same procedure, bearing in mind the necessary adjustments on the model mentioned above, and in this case the values of the viscoelastic parameters were determined with rather low fitting errors. The results are also found in Fig. 6.

\section{DISCUSSION}

From the comparison of the "dispersion relations" obtained from the analysis of the experimental (see Fig. 6) and MD data a great level of agreement follows. This is particularly the case for $Q$-values far from the region where the dispersion reaches its maximum. However, it is not surprising that certain discrepancies appear since it should be recalled that our MD simulation results only apply to centerof-mass motion. One may argue that the reason for such discrepancies is likely to be found in the couplings between rotational degrees of freedom and collective modes. These couplings are not contemplated in the viscoelastic model, originally formulated for monatomic fluids, and imply a certain character of "effectiveness" for the model parameters when applied to a molecular fluid. The similarity of the observed "dispersion relations" with those reported for liquid argon $^{9,10}$ should also be mentioned. In the case of liquid Ar at temperatures well above the melting point a "propagation gap" is readily apparent for $Q$ values around the maximum in the center-of-mass structure factor, $S_{\mathrm{cm}}(Q)$. This "gap" has not been found in the high temperature experiment since $\omega_{0}$ never falls to zero value. If, following the suggestion made by Cohen," the existence of the "gap" in liquid Ar stems from the competition between elastic (restoring) and dissipative forces we would conclude that in the case of $\mathrm{CCl}_{4}$ the former prevail and there exists a continuous propagation of sonic modes through the measured momentum transfer range.

The system does not seem to exhibit any significant change with temperature in "dispersion" data and is in line with the measurements of the static structure factor reported by Misawa, ${ }^{27}$ which shows little variation in the range of temperatures corresponding to our experiment. In this respect, it should be mentioned that no attempt was made to measure spectra at higher temperatures since the decrease in the adiabatic sound velocity and the larger diffusional and rotational contributions would render the separation of single particle and collective effects rather difficult.

The quasilinear behavior exhibited by the low- $Q$ parts obtained for the MD "dispersion relations" can be extrapolated to give the isothermal sound velocity, $c_{l}(Q),{ }^{1}$

$$
L t_{Q \rightarrow 0} \omega_{0}(Q)=c_{t}(Q) Q \text {. }
$$


FIG. 7. (a) Maxwellian relaxation times obtained according to Eq. (7) as a function of the momentum transfer $Q$ for the MD simulation data analysis. Full circles, are the results for the $T=264 \mathrm{~K}$ run and squares are for the $T=293 \mathrm{~K}$ run. (b) Longitudinal viscosities as a function of the momentum transfer for the simulation data. Full circles, are the results for the $T=264$ $\mathrm{K}$ run and squares are for the $T=293 \mathrm{~K}$ run. Intercepts represent the macroscopic values measured for the shear viscosities reported in Ref. 17.

The sound velocity value found at both temperatures agrees well with those deduced from ultrasonic and light scattering measurements as reported by Samios et al. ${ }^{17}$ (1041 and 938 $\mathrm{ms}^{-1}$ at 260 and $293 \mathrm{~K}$, respectively). However, one should bear in mind the fact that at this length scale the sound velocity has become a wave vector dependent quantity and therefore cannot be related to hydrodynamic sound in an easy way.

The relaxation times computed according to Eq. (9) from the viscoelastic parameters are presented in Fig. 7 as a function of the corresponding momentum transfers. A secondary maximum occurs at values of $Q$ corresponding to the secondary minimum in the "dispersion relation," that is for values of $Q$ for which the static structure factor shows its first maximum.

A longitudinal viscosity can be defined from the $\omega_{0}$ and $\omega_{l}$ effective moments in terms of

$$
\eta_{\text {long }}(Q, 0)=\frac{n M}{Q^{2}}\left[\omega_{l}^{2}-\omega_{0}^{2}\right] \tau,
$$

where $n M$ represents the mass density and the derived wavevector-dependent viscosity coefficients are also shown in Fig. 7. As it can be seen from the graph the computed longi- 
tudinal viscosities do not show any noticeable change with the temperature within the momentum-transfer range explored in the present set of experiments. Although the hydrodynamic values for the shear viscosity ${ }^{17}$ could be approached from the low- $Q$ part of the curve, the present data indicate that the characteristic distances (correlation lengths) associated with the referred transport properties are at least one order of magnitude larger than the ones accessible to thermal neutrons. In such a respect, it would be worth estimating the ratio between the hydrodynamic bulk and shear viscosities (not available in the consulted literature), since it may be expected from the data presented above that the bulk-viscosity coefficient should be rather small, so that no pronounced wave vector dependence can be observed for this magnitude. On the other hand, it is well known from ultrasonic ${ }^{28}$ and light scattering studies that the thermal contribution to the Brillouin linewidth cannot be discarded since the relatively large specific heat ratio $(\gamma=1.45)$ will render a heat conduction contribution of the order of $0.068 \mathrm{THz}$ which is not negligible compared with the cited viscous contributions.

On the other hand, the obtained results can be rationalized if one takes into consideration the available ultrasonic studies, ${ }^{28}$ where the sound attenuation coefficient was explained in terms of intermolecular relaxation of the internal vibrational modes. Such a behavior was further confirmed by the lack of any frequency dispersion of the acoustic attenuation up to frequencies of $0.2 \mathrm{GHz},{ }^{29}$ and therefore this explains the absence of noticeable frequency and wave vector dependence for the viscosity coefficient. As a matter of fact, the measured ultrasonic attenuation ${ }^{29}$ gives a coefficient $\alpha / f^{2}=500.10^{-7} \mathrm{~cm}^{-1} \mathrm{~s}^{2}$ which is one order of magnitude higher than the corresponding classical absorption coefficient (i.e., that calculated from shear and heat conduction terms alone) and such an excess absorption has been explained in terms of a collisional mechanism for the lowest lying internal vibrational modes. ${ }^{29}$

An estimation of the limiting $Q$-value signing the change to an overdamped regime can be made from the relationship ${ }^{30}$

$$
3 \omega_{0}^{2}>\omega_{l}^{2}
$$

and it can be easily seen from the graphs that such values are around $0.35 \AA^{-1}$ for the temperatures studied. Therefore, the mode crosses into the nonpropagative regime (overdamped) at nearly the beginning of the explored momentum-transfer range.

A direct comparison with data for liquid argon is precluded by the fact that no equivalent thermodynamical states have been reported. The reduced parameters of state $T^{*}=k_{b} T / \epsilon$ and $n^{*}=n \sigma^{3}$ for the low temperature measurement are $T^{*}=2.82$ and $n^{*}=0.32$, respectively, and the closest set or reported data are given for $T^{*}=2.45$ and $n^{*}=0.19$ which corresponds to a dense-fluid phase and has shown clear inelastic peaks in a recent neutron Brillouin study. ${ }^{15}$ The required number density to match the present observations would be $\sim 8$ atoms $\mathrm{nm}^{-1}$ which would correspond to a pressure 1.6 times higher than the one of $20 \mathrm{MPa}$ reported in Ref. 15. An extrapolation of data given in Fig. 3 of Ref. 15 to the required pressure $(\sim 32 \mathrm{MPa})$ indicates that an inelastic peak somewhat narrower than the one for the $20 \mathrm{MPa}$ case should be observable. Furthermore, the extrapolation of its width to the $Q=0.24 \AA^{-1}$ value corresponding to the lowest wave vector explored in the simulation gives a linewidth of $0.58 \mathrm{THz}$ which compares quite favorably with that depicted in Fig. 4.

\section{v. CONCLUSIONS}

The interplay between computer simulation and inelastic neutron scattering has enabled a consistent analysis of the neutron scattering spectra of liquid carbon tetrachloride. The difficulties found in analyzing this liquid in comparison with some other molecular fluids ${ }^{4,6}$ stems from the fact that exception is made of the lowest achievable wave vectors, the sound mode always exhibits a strong damped character. The spatial dispersion of the excitation shows some characteristics reminiscent of those observed in rare-gas fluids, and shows some indication of negative dispersion as predicted for simple-fluids by some kinetic theory approaches. ${ }^{10} \mathrm{Al}-$ though some alternatives different from the one employed to analyze the data already exist, such as the mode-coupling approach developed by Cohen et al., ${ }^{9}$ we have chosen a simplified phenomenological analysis since as it has been shown by Lovese ${ }^{30}$ the two approaches should be equivalent under certain conditions, and on the other hand, because the former approach requires a larger number of free parameters. ${ }^{9,10}$

A comparison with the spatial dispersion of the collective mode with those already reported for liquids composed of strong polar or hydrogen bonded molecules, ${ }^{5,6}$ reveals that not only do the dispersion curves show a rather different shape but also the wave vector dependence of the damping terms appears to be significatively different.

The collective dynamics liquid carbon tetrachloride can be understood on a qualitative basis, in terms of that corresponding to an L-J liquid properly scaled with the potential parameters. However, even in the hydrodynamic limit, the comparison of several transport properties based upon corresponding states arguments ${ }^{14}$ has been shown to lead to large errors in the prediction of thermodynamical properties from scaling from simple monatomic liquids. In such a respect, it could be worth performing some extension of the principle of corresponding states where the effects of nonsphericity caused by the molecular shapes are explicitly taken into account as a correction term.

It would be worth trying to carry out neutron inelastic measurements as well as simulations in order to cover a broad range of thermodynamic conditions, mainly exploring high pressure and temperature regions, where significant changes in the liquid structure have been reported, ${ }^{27}$ as well as in the disordered (plastic) crystal phase where well defined excitations have been found in a previous computer simulation. ${ }^{13}$

Finally, it should be emphasized the need of a more elaborate formulation of the viscoelastic model where apart from the correlations arising from thermal conduction, the coupling between the heat flux and the momentum current is explicitly taken into account. Such an effect appears to be 
noticeable in this liquid and further progress should be focused onto some phenomenological modeling of it.

\section{ACKNOWLEDGMENTS}

The authors are indebted to Dr. J. Alonso for his guidance during the processing of the MD trajectories data. This work was supported in part by CICYT (Spain) Grant No. PB 890037 C03. Dr. H. Mutka of the I.L.L. is greatly acknowledged for the help given with the preliminary measurements on the IN4 spectrometer. Mr. A. Gomez is kindly acknowledged for the excellent help given during the preparation of the manuscript.

' J. P. Boon and S. Yip, Molecular Hydrodynamics (McGraw-Hill, New York, 1982).

${ }^{2}$ J. R. D. Copley and S. W. Lovesey, Rep. Prog. Phys. 38, 461 (1975).

${ }^{3}$ See, for instance the pioneering works on liquid metals by J. R. D. Copley and J. M. Rowe, Phys. Rev. Lett. 32, 49 (1974). For seminal works on rare-gases, see C.-H. Chung and S. Yip, Phys. Lett. A 50, 175 (1974), and references given in Ref. 10.

${ }^{4}$ F. J. Bermejo, J. L. Martinez, D. Martin-Marero, F. J. Mompean, M. Garcia-Hernandez, and A. Chahid, Phys. Lett. A 158, 253 (1991).

${ }^{5}$ F. J. Bermejo, F. Batallan, E. Enciso, M. Garcia-Hernandez, J. Alonso, and J. L. Martinez, Europhys. Lett. 12, 129 (1990); F. J. Bermejo, R. Ramirez, J. L. Martinez, C. Prieto, F. Batallan, and M. Garcia-Hernandez, J. Phys. Condensed Matter 3, 569 (1991).

${ }^{6}$ F. J. Bermejo, J. L. Martinez, D. Martin, M. Garcia-Hernandez, F. J. Mompean, and J. Alonso, J. Chem. Phys. 95, 5387 (1991).

'F. J. Bermejo, J. L. Martinez, D. Martin Marero, F. Mompean, M. Garcia-Hernandez, and A. Chahid, Physica B (in press).

${ }^{8}$ J. L. Martinez, F. J. Bermejo, M. Garcia-Hernandez, and F. J. Mompean, J. Phys. Condensed Matter 3, 3849 (1991).
'E. G. D. Cohen, I. M. de Schepper, and M. J. Zuilhof, Physica B and C 127, 282 (1984), and references therein.

${ }^{10}$ L. A. de Graaf, in Static and Dynamic Properties of Liquids, Vol. 40 in Springer Proceedings in Physics, edited by M. Davidovic and A. K. Soper (Springer, Berlin, 1990), also references therein.

${ }^{11}$ F. J. Bermejo, M. Alvarez, M. Garcia-Hernandez, F. Mompean, R. P. White, W. S. Howells, C. J. Carlile, E. Enciso, and F. Batallan, J. Phys. Condensed Matter 3, 851 (1991).

${ }^{12}$ A. Chahid, F. J. Bermejo, J. L. Martinez, and E. Enciso, J. Phys. Condensed Matter 4, 1213 (1992).

${ }^{13}$ I. R. McDonald, D. G. Bounds, and M. L. Klein, Mol. Phys. 45, 521 (1982).

${ }^{14}$ J. Rouch, J. P. Boon, and P. A. Fleury, Physica A 88, 347 (1977).

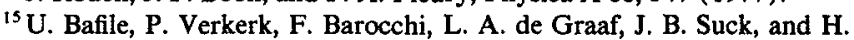
Mutka, Phys. Rev. Lett. 65, 2394 (1990).

${ }^{16}$ Guide to Neutron Research Facilities at ILL, edited by H. Blank and B. Maier (Grenoble, France, 1988).

${ }^{17}$ D. Samios and Th. Dorfmuller, Mol. Phys. 41, 637 (1980).

${ }^{18}$ O. Söderstrom, J. R. D. Copley, J. B. Suck, and B. Dorner, J. Phys. F 10, L151 (1980).

${ }^{19}$ M. Johnson, AERE Report No. 7682.

${ }^{20} \mathrm{~J}$. Alonso, M. Garcia-Hernandez, F. J. Bermejo, and J. L. Martinez, ILL Internal Report No. 91AL01T.

${ }^{21}$ Program RESCAL, ILL software.

${ }^{22}$ D. Gorlitz, J. Kotzler, F. J. Bermejo, P. Boni, and J. L. Martinez, Physica $B$ (in press).

${ }^{23} \mathrm{~F}$. Rieutord (personal communication).

${ }^{24}$ S. M. Thompson, Program MoteTrA, from CCP5 Program Library, Daresbury Laboratory.

${ }^{25}$ J. P. Hansen and I. R. McDonald, in Theory of Simple Liquids (Academic, New York, 1986).

${ }^{26} \mathrm{~S}$. W. Lovesey, Theory of Neutron Scattering from Condensed Matter (Oxford University, Oxford, 1986).

${ }^{27}$ M. Misawa, J. Chem. Phys. 91, 5649 ( 1989); F. J. Bermejo, E. Enciso, J. Alonso, N. Garcia, and W. S. Howells, Mol. Phys. 64, 1169 (1988).

${ }^{28}$ K. F. Herzfeld, J. Acoust. Soc. Am. 13, 33 (1941).

${ }^{29}$ K. F. Herzfeld, J. Acoust. Soc. Am. 29, 1180 (1957).

${ }^{30}$ S. W. Lovesey, Z. Phys. B 58, 79 (1985). 